\title{
EVOLUTION OF ANAESTHESIA MANAGEMENT FOR TRANSFEMORAL AORTIC VALVE IMPLANTION IN AORTIC STENOSIS. REVIEW OF CASES OF OUR CENTER
}

\author{
B. Elena ${ }^{1}$, M. Joaquin ${ }^{1}$, G. Laura ${ }^{1}$, M. Alba ${ }^{1}$, F. Clara ${ }^{1}$, D. A. José ${ }^{1}$ \\ ${ }^{1}$ hospital general - VALENCIA (Spain)
}

\section{Background and Goal of Study}

Transcatheter aortic valve implantation (TAVI) is emerging as an alternative, less invasive technique for aortic stenosis.

The tendency to minimal invasion has led to a modification of the anesthetic technique.

We review the anesthetic management of TAVIs performed at the General Hospital of Valencia from October 2012 to the present.

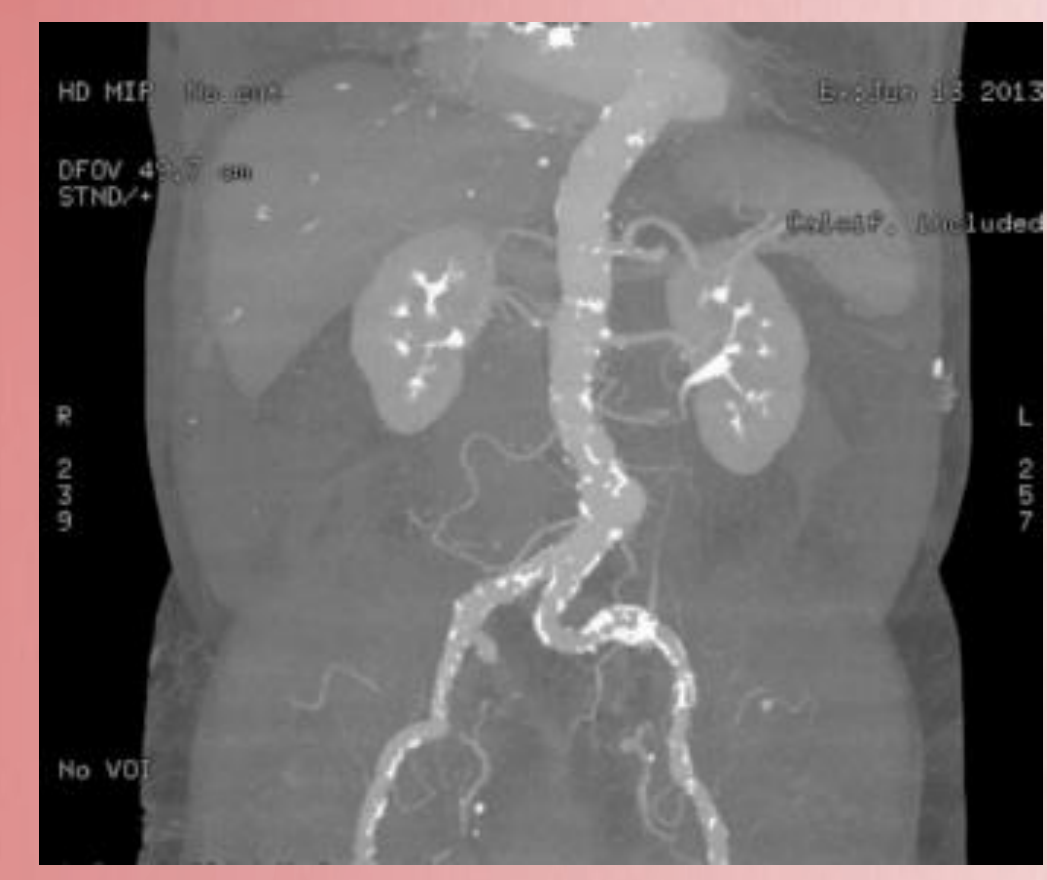

\section{Results and Discussion:}

The anesthetic technique of choice in TAVI remains a matter of discussion. Sedation reduces time and costs, as well as allowing neurological monitoring. Note the role of the LMA because it avoids laryngoscopy and intubation, as well as the use of neuromuscular blockers allowing an early awakening. It is common that at the beginning of the learning curve, in more complicated procedures or in certain groups with more risk of complications (difficult airway, obstructive sleep apnea), an AG + $E T I$ is chosen and that the use of the ML and sedation in those cases indicate.

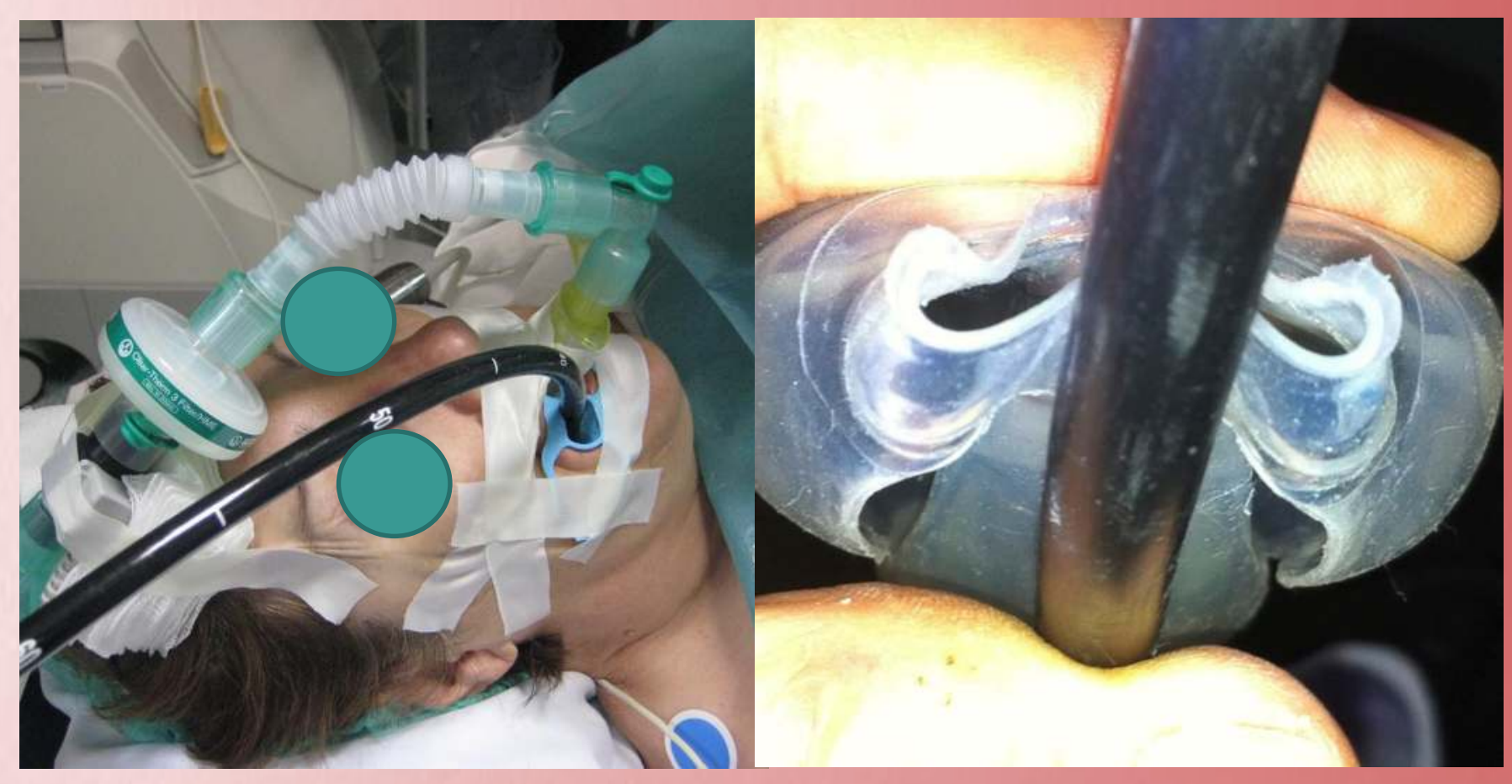

\section{Materials and Methods:}

Initially, the transfemoral TAVI was performed under fluoroscopic and echocardiographic control, the anesthetic technique being general anesthesia (GA) with endotracheal intubation (ETI). Since October 2015 the use of GA with laryngeal mask (LMA) (18 cases) and sedation (2) has been progressively introduced, coinciding with the abandonment of TEE and improved learning curve by hemodynamics.

\section{Conclusion(s):}

Despite the evolution in the learning curve of the responsible team, complications of significant repercussions sometimes arise. Therefore, the choice of anesthetic technique should be based on patient comorbidity, institutional practices and team experience. 